New Solution to High-Field Transport in Semiconductors:

I. Elastic Scattering without Energy Relaxation

\title{
Kenji Natori*
}

Institute of Applied Physics, University of Tsukuba, Tsukuba, Ibaraki 305-8573, Japan

High-field transport in a semiconductor diode structure at room temperature is newly analyzed in a reflection-transmission regime. The Boltzmann equation with a constant electric field $E$ is applied to a semiconductor channel of length $L$, and a pair of flux equations is analytically solved. For applied fields larger than $\sim 500 \mathrm{~V} / \mathrm{cm}$ for silicon, the energy relaxation by the acoustic phonon scattering hardly catches up with the energy gain from the field, and the kinetic energy accumulates generating hot carriers. As a limiting case, in this paper we analyze carrier transport with elastic scattering and without energy relaxation. Current density proportional to $\sqrt{E / L}$ is derived for a wide electric field range. At much higher fields, the ballistic transmission of carriers through the channel occurs, and current density tends to saturate. The result will find its application in sufficiently small systems, where energy relaxation by optical phonon scattering is not dominant.

\footnotetext{
*E-mail address: natori@esys.tsukuba.ac.jp
} 


\section{Introduction}

The high-field transport of semiconductors is an old topic, and yet its importance is still increasing. A half century ago, Ryder ${ }^{1)}$ and Shockley ${ }^{2)}$ investigated the current in germanium and silicon, and proposed the theory of the drift-diffusion current ${ }^{3)}$ as well as the velocity saturation. Since then, the theory has served as the central concept of semiconductor electronics. Now that devices are in nanoscale and the electric field inside is intensified, proper understanding of the mechanism of high-field transport is critical for the control of device performance. Here we present a new solution of high-field transport based on analysis of the Boltzmann transport equation (BTE) ${ }^{4)}$ in the reflection transmission regime.

Analysis of BTE is an orthodox approach to the classical transport theory. The one-dimensional $\mathrm{BTE}^{5-7)}$ is also frequently analyzed to elucidate essential features of a transport system. The phenomenological relaxation time approximation (RTA) is usually assumed. In the 1960s, a numerical approach to solving BTE using the Monte Carlo technique $^{8-10)}$ was developed and has become one of the standard methods for the numerical simulation of transport in semiconductors. It has been a very efficient method of analysis. However, the numerical method sometimes shows weakness in developing physical concepts. The reflection-transmission regime, which was introduced by Landauer's formula, ${ }^{11,12)}$ had a profound effect on the transport theory. In place of the borderless homogeneous system, a finite-size system equipped with a current source and drain electrodes is assumed, and the transmission of a flux from the source to the drain is analyzed. The flux theory proposed by McKelvey's group ${ }^{13-16)}$ is an example of the classical application of the regime.

The drift current density $I$ under a constant electric field $E$ is usually described as Ohm's law. $^{17)}$ 


$$
I=\frac{n q^{2} \tau}{m} E
$$

Here, $q, m$, and $n$ are the charge, the effective mass, and the density of carriers, respectively. $\tau$ is discussed later. Equation (1) is often divided into $I=q n v$ and $v=(q \tau / m) E$ where $v$ is the drift velocity. This is the core expression of the drift-diffusion model. The current density at a local point is expressed by only the local values of parameters. It is uniform in a homogeneous system. Although a similar expression is readily derived by a heuristic approach of the one-particle Drude theory, ${ }^{18)}$ BTE provides a more rigorous derivation of eq. (1) for a homogeneous bulk system considering the carrier distribution. The most remarkable assumption used in deriving eq. (1) is RTA., ${ }^{3,4,19)}$ The complicated collision integral of BTE is replaced by a simple expression,

$$
\left(\frac{\partial f_{\mathbf{k}}}{\partial t}\right)_{\text {coll. }}=-\frac{1}{\tau}\left(f_{\mathbf{k}}-f^{0} \mathbf{k}\right),
$$

where $f_{\mathbf{k}}\left(f_{\mathbf{k}}^{0}\right)$ is the distribution function with the applied field (in thermal equilibrium). The deviation of carrier distribution from the equilibrium is assumed to relax to the equilibrium in the relaxation time $\tau$ [the same $\tau$ as in eq. (1), the averaged value over the Fermi surface when the carrier distribution is considered]. RTA is generally thought to be effective when the deviation is small. For a large deviation in contrast, it seems difficult to justify such a simple assumption. Details of the scattering process, or the complicated courses of energy relaxation satisfying the conservation rules, are all neglected. The interplay of carriers through the scattering between different $k$-states is replaced by a simple self-relaxing process. It is a sort of mean field theory neglecting details. This is a drastic approximation, but it is almost inevitable in an analytic approach. 
Equation (1) describes the drift motion of carriers driven by the electric field. Carriers gain kinetic energy from the field as they drift downstream, and relax the energy to the phonon system. At a low electric field where the energy gain is not significant, carriers can exhaust the gained energy through subsequent energy relaxation (presumably owing to the acoustic phonon scattering). The increase in mean kinetic energy is suppressed, and the linear E-dependence of $I$ is maintained. Equation (1) assumes that carriers stay in a homogeneous quasi-equilibrium around each local point, fully relaxing the gained energy to phonon system (full-energy-relaxation model). The equation is usually derived by applying BTE to a borderless uniform system. ${ }^{4,19)}$ On the other hand, Appendix A shows how the full-energy-relaxation model derives the drift-diffusion current, and that the result is valid in the low-field range $E \leq 490 \mathrm{~V} / \mathrm{cm}$ for silicon.

For $E \geq 500 \mathrm{~V} / \mathrm{cm}$ in contrast, the gained energy cannot be exhausted by subsequent scatterings. The mean kinetic energy of carriers increases generating hot carriers. ${ }^{20,21)}$ The carrier velocity increases as they drift downstream. The uniformity of the system, that is, the theoretical background of eq. (1), collapses. According to the conventional theory, the hot carriers demand modification of the relaxation time $\tau$, and attain a new quasi-equilibrium, which is also expressed by eq. (1). The increase in mean kinetic energy modifies the mean scattering probability, and causes an E-dependent mobility. The current expression includes higher-order terms, constituting a perturbation expansion in powers of E. Shockley also derived $^{2)}$ a current expression proportional to $\sqrt{E}$ using eq. (1) for a high field of $E \geq 1.5 v_{s} / \mu_{0} \quad\left(\approx 800 \mathrm{~V} / \mathrm{cm}\right.$ for silicon; here, $v_{\mathrm{s}}$ is the sound velocity and $\mu_{0}$ is the low-field mobility). However, the clear dependence on $\sqrt{E}$ was not confirmed in experiments ${ }^{21)}$. The E-linearity of current density loses its foundation in the high-field range of $E \geq \sim 500 \mathrm{~V} / \mathrm{cm}$. 
On the other hand, the experimental data suggest an approximate linearity in $I-E$ relationship extending into higher fields less than the velocity saturation limit. What is the mechanism supporting the linearity at high fields?

In present analysis, we propose a new solution of high-field transport based on a pseudo-one-dimensional BTE ${ }^{22,24)}$ and try to clarify the transport mechanism in the high-field region where the conventional eq. (1) is left groundless. The two major assumptions in eq. (1), RTA and perturbation expansion, are removed, and the BTE is solved yielding a consistent solution from low fields to high fields. In Part I, the carrier transport only with the elastic scattering and in the absence of energy relaxation is analyzed. In Part $\mathrm{II},{ }^{24)}$ we investigate the high-field transport in which the elastic scattering and also the energy relaxation due to optical phonon scattering are considered. In $\S 2$, we analyze the transport associated with elastic scattering without energy relaxation. Section 3 is devoted to the discussion of transport mechanism in the transmission regime, and $\S 4$ is a summary.

\section{Model 1: Elastic Scattering Without Energy Relaxation}

We investigate carrier transport in a semiconductor in the reflection-transmission probability regime for a room-temperature nondegenerate case. The carrier transport through the region (referred to hereafter as the channel) of length $L$, interposed between the source electrode grounded and the drain electrode biased at $V$, is analyzed assuming that a constant electric field $E=V / L$ is applied. The device structure in the transverse direction is uniform. The source and drain electrodes are assumed to be ideal reservoirs of carriers. The flux of the carriers they emit is as large as the channel requires, and they accept flux as the channel discharges without reflection. For simplicity, positive charge carriers are assumed and we 
suppose $q>0$. The result is also applied to electronic current. We may need to change the signs of some quantities, but the magnitudes are unaltered and no confusion is caused. The $x$-axis is along the channel toward the drain, and the origin is at the source edge. The field is uniform along the $x$-axis, and the system is homogeneous in the $y$-z-plane and has an axial symmetry around the $x$-axis.

As was mentioned above, the full-energy-relaxation model derives the drift-diffusion theory. In a higher-field range in contrast, the kinetic energy of carriers accumulates during the course of their transport toward the drain. To study this situation opposite to the full-energy-relaxation, we analyze an extreme case in which only elastic scattering without energy relaxation is assumed. Figure 1 schematically shows a potential diagram of the device. The classical BTE approach is employed. We try to analyze the system without RTA by virtue of a slightly stronger restriction than is usually employed in elastic scattering. Elastic scattering conserves the total carrier energy, and the transverse kinetic energy perpendicular to the field increases or decreases by chance. Regarding the chance of an increase as even to that of a decrease, we tried assuming that the transverse kinetic energy is conserved in the process on average. On the basis of this assumption, we can transform the three-dimensional BTE to a steady-state pseudo-one-dimensional BTE, as is detailed in Appendix B. A discussion on the effect of the failure of this assumption is found at the end of $\S 3$.

As in Fig. 1, carriers are assumed to be injected from the source to the channel with the kinetic energy $\varepsilon$, which is on the order of thermal energy $k_{\mathrm{B}} T$, and are transferred along the channel, being accelerated by electric field and suffering from elastic scattering. The origin in the $x$-axis is at the source edge, and the origin in energy is at the potential energy level at $x=0$. In the pseudo-one-dimensional BTE approach, the two-dimensional phase space 
composed of the wave number $k$ and the position $x$ is assumed. Owing to energy conservation in the direction of the field, the kinetic energy of a carrier at the position $x$ with the wave number $k$ is $(\hbar k)^{2} / 2 m=(q E x+\varepsilon)$ and depends on the position $x$. Here, $\hbar$ is the reduced Planck's constant. The $k-x$ relation defines a parabola in the phase space, and the carrier motion is confined within this track. The state of a carrier in the channel is classified into the positive velocity state $(k>0)$ and the negative velocity state $(k<0)$. According to the model in Appendix $\mathrm{B}$, the carrier scattering at $x$ is allowed only between the states $k$ and $-k$. The scattering probability from $k$ to $-k$ is equal to that from $-k$ to $k$ by the principle of detailed balancing. The steady-state pseudo-one-dimensional BTE derived in eq. (B6) is

$$
\frac{q}{\hbar} E \frac{\partial f(x, k)}{\partial k}+\frac{\hbar k}{m} \frac{\partial f(x, k)}{\partial x}+B[f(x, k)-f(x,-k)]=0 .
$$

Here $f(x, k)$ is the pseudo-one-dimensional distribution function defined in eq. (B7) (the dimension is $\left.\left[\mathrm{L}^{-2}\right]\right) . B$ is the backscattering probability between the states $k$ and $-k$, and is given by $B=1 / 2 \tau_{e}$ where $\tau_{\mathrm{e}}$ is the three-dimensional elastic scattering time. The energy dependence of $B$ is neglected for simplicity. Since the total energy of a carrier is conserved, the distribution function $f(x, k)$ is expressed by a form including the delta function, assuring energy conservation. If we prepare appropriate functions $f^{*}(x,|k|)$ where $|k|=\sqrt{2 m(q E x+\varepsilon)} / \hbar$, and $F(x)$, the positive-velocity component is expressed as

$$
\begin{array}{r}
f(x,|k|) \equiv f^{*}\left(x, \frac{\sqrt{2 m(q E x+\varepsilon)}}{\hbar}\right) \delta\left(\frac{\hbar^{2} k^{2}}{2 m}-q E x-\varepsilon\right) \\
\equiv F(x) \delta\left(\frac{\hbar^{2} k^{2}}{2 m}-q E x-\varepsilon\right) .
\end{array}
$$

Similarly, the negative-velocity component is expressed by an appropriate function $G(x)$ 


$$
f(x,-|k|) \equiv G(x) \delta\left(\frac{\hbar^{2} k^{2}}{2 m}-q E x-\varepsilon\right) .
$$

After substituting eqs. (4a) and (4b) into eq. (3) followed by appropriate rearrangement, we integrate the resultant expression over $k$ to eliminate the $\delta$-function. Device experts may not be familiar with the $\delta$-function. Refer to the following formula if necessary. If the equation $g_{1}(x)=0$ has a unique solution $x=x_{0}$ within an appropriate range $x_{1}<x<x_{2}$, then,

$$
\int_{x_{1}}^{x_{2}} g_{2}(x) \delta\left[g_{1}(x)\right] d x=\frac{g_{2}\left(x_{0}\right)}{g_{1}{ }^{\prime}\left(x_{0}\right)}
$$

Thus, we obtain a set of differential equations that $F(x)$ and $G(x)$ are required to satisfy.

$$
\begin{aligned}
& \sqrt{\frac{2(q E x+\varepsilon)}{m}} \frac{d F}{d x}+B(F-G)=0, \\
& -\sqrt{\frac{2(q E x+\varepsilon)}{m}} \frac{d G}{d x}+B(G-F)=0 .
\end{aligned}
$$

Using eqs. (4a) and (4b), we derive the carrier fluxes for the positive- and negative-velocity components at $x$, respectively, as in eq. (B9):

$$
\begin{aligned}
& \int_{0}^{\infty} f(x, k) \frac{\hbar k}{m} \frac{d k}{2 \pi}=\frac{1}{h} F(x), \\
& \int_{-\infty}^{0} f(x, k) \frac{\hbar k}{m} \frac{d k}{2 \pi}=-\frac{1}{h} G(x) .
\end{aligned}
$$

$h$ is Planck's constant. Functions $F(x)$ and $G(x)$ divided by $h$ represent the magnitude of three-dimensional fluxes. Equations (5a) and (5b) divided by the square root factor representing the carrier velocity on the left-hand side, imply the continuity of each velocity component of carriers, because the flux divided by the velocity gives the carrier density of each component. The three-dimensional carrier density $n_{\varepsilon}(x)$ for the incident energy $\varepsilon$ is obtained [eq. (B8)] as 


$$
n_{\varepsilon}(x)=\int_{-\infty}^{\infty} f(x, k) \frac{d k}{2 \pi}=\frac{\sqrt{m}}{\sqrt{2} h} \frac{F(x)+G(x)}{\sqrt{q E x+\varepsilon}} .
$$

Using the relation $\sqrt{2(q E x+\varepsilon) / m}(d / d x)=(q E / \sqrt{2 m})(d / d \sqrt{q E x+\varepsilon})$, we easily integrate eqs. (5a) and (5b) to obtain the general solution. The specific solution within the channel for the boundary condition $F(0)=F_{0}$ as well as $G(L)=G_{\mathrm{L}}$, as in Fig. 1, is derived as

$$
\begin{aligned}
& F(x)=\frac{\left\lfloor q E+\sqrt{2 m B^{2}}(\sqrt{q E L+\varepsilon}-\sqrt{q E x+\varepsilon})\right] F_{0}-\sqrt{2 m B^{2}}(\sqrt{\varepsilon}-\sqrt{q E x+\varepsilon}) G_{L}}{q E+\sqrt{2 m B^{2}}(\sqrt{q E L+\varepsilon}-\sqrt{\varepsilon})} \\
& G(x)=\frac{\sqrt{2 m B^{2}}(\sqrt{q E L+\varepsilon}-\sqrt{q E x+\varepsilon}) F_{0}+\left[q E-\sqrt{2 m B^{2}}(\sqrt{\varepsilon}-\sqrt{q E x+\varepsilon})\right] G_{L}}{q E+\sqrt{2 m B^{2}}(\sqrt{q E L+\varepsilon}-\sqrt{\varepsilon})} .
\end{aligned}
$$

The transmission coefficient $\bar{T}$ from the source to the drain is obtained as the value $F(L) / h$ for the boundary conditions $F_{0}=h$ and $G_{L}=0$, and has the form

$$
\bar{T}=\frac{q E}{q E+\sqrt{2 m B^{2}}(\sqrt{q E L+\varepsilon}-\sqrt{\varepsilon})} .
$$

For carriers moving in the opposite direction, from the drain to the source, the transmission coefficient has the same expression. In the limit of $E \rightarrow 0$, the transmission coefficient for the diffusion without the field is reduced to

$$
\bar{T}_{E \rightarrow 0}=\frac{\sqrt{2 \varepsilon}}{\sqrt{2 \varepsilon}+\sqrt{m B^{2}} L}=\frac{\ell}{\ell+L},
$$

where $\ell$ is the mean free path given by the carrier velocity $\sqrt{2 \varepsilon / m}$ multiplied by the scattering time $1 / B$. Note that this expression agrees with the formula in the one-dimensional multi-scattering theory derived by Datta ${ }^{11)}$.

The current density $I_{\varepsilon}$ from the source to the drain attributable to the carriers with the incident energy $\varepsilon$ is derived from eqs. (8a) and (8b) as 


$$
I_{\varepsilon}=\frac{q}{h}[F(x)-G(x)]=\frac{q}{h} \bar{T}\left(F_{0}-G_{L}\right)
$$

It is a constant along $x$ due to the current conservation for the case without energy relaxation. The current vanishes if $F_{0}=G_{L} \cdot F_{0}$ and $G_{\mathrm{L}}$ are injected from the source and drain electrodes, respectively. We assume that the source and the drain are in quasi-equilibrium with the Fermi potentials $\mu$ and $(\mu-q V)$, respectively, referring to the origin of energy in Fig. 1. More specifically, we assume that the source and the drain, both made of highly doped semiconductors with degenerate carriers, are connected to a semiconductor channel region with a uniform doping concentration $n_{0}$ via a low-resistance junction of the type $\mathrm{n}^{+}-\mathrm{n}$ or $\mathrm{p}^{+}-\mathrm{p}$, which is denoted hereafter by the source- or drain-channel junction. The entrance (exit) part of the channel neighboring the source (drain) is in quasi-equilibrium with the electrodes, and the Fermi potential therein is adjusted to a value close to $\mu(\mu-q V)$ by the potential barrier across the source-channel (drain-channel) junction. The potential profile across the structure is provided by the solution of the Poisson equation self-consistently coupled to the charge distribution. Within the bulk region of the channel, the constant field profile is assured if charge neutrality therein is maintained. The entrance (exit) part of the channel is in quasi-equilibrium with the source (drain) controlled by Maxwell-Boltzmann statistics, when the injection level of carriers is not so high. The majority carrier density therein is about $n_{0}$, the doping level of the channel, and here we assume the value. The carrier distribution function in the entrance part for example is approximated by eq. (B11) and has a continuous distribution in energy. The flux injected into the channel is the superposition of the contribution from each energy component as is given in eq. (B13). In case the distribution function has a monochromatic energy component as in eq. (4), current density is derived as in 
eq. (11) and is linear in the injected fluxes $F_{0} / h$ and $G_{\mathrm{L}} / h$. When the energy spectrum has a distribution, the current density is obtained by superposition of eqs. (11) in which $F_{0} / h$ and $G_{\mathrm{L}} / h$ are replaced by various flux components of eq. (B13) with different $\varepsilon_{\mathrm{x}}$. The injection from the entrance part is expressed by the substitution equation,

$$
\frac{F_{0}}{h} \times \rightarrow \int d \varepsilon \frac{n_{0}}{\sqrt{2 m \pi k_{B} T}} \exp \left(-\frac{\varepsilon}{k_{B} T}\right) \times
$$

The injection from the drain, $G_{L} / h$, is also expressed by the substitution eq. (12), but the factor $\exp \left(-q V / k_{B} T\right)$ is multiplied in this case. If we integrate over energy the flux component in eq. (12) divided by the carrier velocity $\sqrt{2 \varepsilon / m}$, we obtain the carrier density of the injected flux as $n_{0} / 2$, the density of the positive-velocity carriers. The injection velocity, which is the mean velocity of injected carriers, is obtained by integrating eq. (12) and then dividing it by $n_{0} / 2$ to yield $\sqrt{2 k_{B} T / \pi m}$, the thermal velocity for the positive velocity component.

Substituting for $F_{0} / h$ and $G_{L} / h$ in eq. (11) in accordance with the substitution eq. (12), we obtain the total current density I of the system,

$$
I=\frac{q n_{0}}{\sqrt{2 m k_{B} T \pi}}\left[1-\exp \left(-\frac{q V}{k_{B} T}\right)\right] \int_{0}^{\infty} \exp \left(-\frac{\varepsilon}{k_{B} T}\right) \bar{T} d \varepsilon .
$$

We next try to estimate some quantities numerically and discuss the transport of silicon. The low field mobility $\mu$ of the high-purity silicon is reported to be $1430 \mathrm{~cm}^{2} \mathrm{~V}^{-1} \mathrm{~s}^{-125)}$, and we estimate the three-dimensional elastic scattering time $\tau_{\mathrm{e}}=2 \times 10^{-13} \mathrm{~s}$ in view of the relation $\mu=q \tau_{e} / m . B=\left(1 / 2 \tau_{e}\right)$ is estimated to be $2.5 \times 10^{12} \mathrm{~s}^{-1}$. The spherical conductivity effective mass for silicon is assumed to be $m=\left[\left(2 m_{t}^{-1}+m_{\ell}^{-1}\right) / 3\right]^{-1}=0.26 m_{0}$, where $m_{0}$ is the electron mass. Figure 2 shows the distribution of the carrier density $n_{\varepsilon}(x)$ within the region $x=0-2.0 \mu \mathrm{m}$ for $\varepsilon=0.026 \mathrm{eV}$, comparable to the thermal energy at room temperature. The 
electric field is $E=1000 \mathrm{~V} / \mathrm{cm}$. The ordinate is normalized by the density of injected carriers, $\left(F_{0} / h\right) \sqrt{m / 2 \varepsilon}$. The injection from the drain side by $G_{\mathrm{L}}$ is neglected owing to the assumption that $q V>>k_{B} T . L$ is varied from 0.2 to $20 \mu \mathrm{m}$ as the parameter. The curves show how injected carrier density evolves along the channel. We can see that the density sharply decreases toward the drain, in contrast to the ordinary drift current. The density decrease along the channel varies depending on $L$. The normalized carrier density 2.0 at $x=0$ implies that the large positive- and negative-velocity fluxes almost balance each other to yield a small net current. Carriers moving toward the drain are disturbed by perpetual backscattering, which drives them back. The number of carriers that survive the backscattering and advance toward the drain diminishes as they near the drain. As a result, the carrier flow is stagnant close to the source. Figure 3 shows plots of the drift velocity $v_{\varepsilon}(x)$ normalized by the velocity of injected carriers, $\sqrt{2 \varepsilon / m}$, which is on the order of thermal velocity. We assume $G_{L}=0, \varepsilon=0.026 \mathrm{eV}$, and $E=1000 \mathrm{~V} / \mathrm{cm} . L$ is varied as a parameter. We can see that the drift velocity of the injected carriers increases along the $x$-axis, in contrast to the expected constant velocity of the drift-diffusion theory. A curve of constant velocity given by $\left(q \tau_{e} / m\right) E$ is also illustrated. Carrier density decreases toward the drain, and the conservation of electric current naturally causes an increase in drift velocity toward the drain. Figure 4 shows plots of the transmission coefficient $\bar{T}$ as a function of the electric field $E$. The parameter is $L . \bar{T}$ increases as $E$ increases, and approaches unity as $E \rightarrow \infty$, yielding the total (ballistic) transmission, as is inferred in eq. (9). Carriers are instantaneously accelerated and reach the drain before backscattering takes place. Therefore, all carriers injected at the source are transmitted to the drain. On the other hand, $\bar{T}$ decreases as $L$ increases, and it approaches zero as $L \rightarrow \infty$, as is also inferred in eq. (9). 
Figure 5 shows plots of the total current density $I$ in eq. (13) at $T=300 \mathrm{~K}$ as a function of $E$. Here, we assume that the doping level is $n_{0}=2.5 \times 10^{14} \mathrm{~cm}^{-3}(\sim 28 \Omega \mathrm{cm}$ in $n$-type silicon $)$ in view of the later discussion in Part II. $L$ is varied as a parameter. For a sufficiently small value of $E$, we can recognize a region where $I$ is proportional to $E$ and is almost independent of $L$. As the field $E$ increases, the proportionality to $E$ breaks down and the slope of the curve in the figure shows the dependence $I \propto \sqrt{E}$ for a wide region of $E$. In this region, $I$ shows a strong dependence on $L$ and decreases as $L$ increases. The drift-diffusion current at a local point $x$ is known to be a function of local parameters: local carrier density and local drift velocity dependent on local scattering time and local field. However, the $L$ dependence in Fig. 5 shows that the current is not local but is dependent on the global size of the system.

\section{Discussion}

Analysis of eq. (13) by deriving asymptotic expressions helps clarify the transport mechanism. First, we find the leading term in $E$ in the low-field region, $E<<k_{B} T / q L$. The factor in the central bracket on the right-hand side of eq. (13) is reduced to $q E L / k_{B} T$. The leading contribution of the transmission coefficient $\bar{T}$ in the integral is the term of the zero-th order in $E$, which is only eq. (10). If we assume a system size of $L \sim 1 \mu \mathrm{m}$ or larger, and $\varepsilon \sim k_{B} T$, then $\sqrt{m B^{2}} L \geq 3.0(\mathrm{eV})^{0.5}>>\sqrt{2 \varepsilon} \approx 0.23(\mathrm{eV})^{0.5}$ (this implies that $L>>\ell$ ), and thus eq. (10) is reduced to $\bar{T} \approx \sqrt{2 \varepsilon /\left(m B^{2} L^{2}\right)}=\ell / L$. Substituting into eq. (13) and integrating over $\varepsilon$, we obtain the current density

$$
I \approx \frac{q^{2} n_{0}}{2 m B} E, \quad\left(E<<\frac{k_{B} T}{q L}\right) .
$$

This is the same as eq. (1), but the validity is limited to a very low applied field less than 
$k_{B} T / q L$.

In the field region of $k_{B} T / q L<<E<<2 m B^{2} L / q$, the factor in the central bracket in eq. (13) is reduced to unity and is independent of $E$. Now we define the traverse time $\Delta t_{x_{1} \rightarrow x_{2}}^{\varepsilon}$ of a positive-velocity carrier with the incident energy $\varepsilon$ at $x=0$, from point $x_{1}$ to point $x_{2}$, in the absence of scattering. Newton's equation of a carrier for a constant acceleration by the electric field $E$ yields

$$
\Delta t_{x_{1} \rightarrow x_{2}}^{\varepsilon}=\frac{\sqrt{2 m}\left(\sqrt{\varepsilon+q E x_{2}}-\sqrt{\varepsilon+q E x_{1}}\right)}{q E} .
$$

We can assume $\varepsilon \sim k_{B} T$ and we have $\Delta t_{0 \rightarrow L}^{\varepsilon}=\sqrt{2 m}(\sqrt{q E L+\varepsilon}-\sqrt{\varepsilon}) / q E \approx \sqrt{2 m L / q E}$ if the incident kinetic energy $\varepsilon$ is sufficiently less than $q E L$. Using the collision (backscattering) time $\tau_{c}=1 / B$, we transform the inequality $E<<2 m B^{2} L / q$ to $\Delta t_{0 \rightarrow L}^{\varepsilon} \gg \tau_{c}$, which implies that the carrier would suffer many collisions before reaching the drain. The transmission coefficient in eq. (9) is rewritten, and is also approximated by

$$
\bar{T}=\frac{\tau_{c}}{\tau_{c}+\Delta t_{0 \rightarrow L}^{\varepsilon}} \approx \frac{\tau_{c}}{\Delta t_{0 \rightarrow L}^{\varepsilon}} \approx \sqrt{\frac{q E}{2 m B^{2} L}},
$$

where the last term is independent of $\varepsilon$. After integration over $\varepsilon$, we obtain

$$
I \approx q^{3 / 2} n_{0} \frac{\sqrt{k_{B} T}}{2 \sqrt{\pi} m B} \sqrt{\frac{E}{L}} \quad\left(k_{B} T / q L<<E<2 m B^{2} L / q\right),
$$

which is proportional to $\sqrt{E}$ and dependent on $L$, as is expected. Note that the second half of eq. (10) is equivalent to the first half of eq. (16) provided that $E=0$; eq. (16) also covers the case $E \neq 0$. One may remember that Shockley ${ }^{2)}$ derived a similar current expression proportional to $\sqrt{E}$. Note that eq. (17) is completely different from his discussion. His theory was based on energy dissipation to a phonon system, and the result showed no 
dependence on the sample size $L$.

For a much larger field, the condition $2 m B^{2} L / q<<E$ is reduced to $\Delta t_{0 \rightarrow L}^{\varepsilon}<<\tau_{c}$, which means that the mean distance of the course that the carrier runs accelerated by the field during the scattering time is much larger than $L$. Then, the transmission coefficient in eq. (16) is reduced to unity (ballistic transmission without backscattering), and we obtain

$$
I=q \frac{n_{0}}{2} \sqrt{\frac{2 k_{B} T}{\pi m}} \quad\left(2 m B^{2} L / q<<E\right)
$$

This implies that the carriers with the density $\mathrm{n}_{0} / 2$ (the positive velocity component) are injected from the source into the channel with the velocity $\sqrt{2 k_{B} T / \pi m}$ (the thermal velocity of the positive-velocity carriers regulated by Boltzmann distribution), and are then completely transmitted to the drain without backscattering. The current shows no dependence on $E$ and saturates. However, the required field is exceedingly high, as the transmission coefficient shown in Fig. 4 suggests, unless the channel length $L$ is very small.

The carrier density distribution shown in Fig. 2 suggests a marked deficit of carriers in the channel. Let us examine the mean carrier density $\langle n\rangle \equiv(1 / L) \int_{0}^{L} n(x) d x$ inside the channel. The carrier density $n(x)$ for eq. (13) is obtained by substituting eqs. (8a) and (8b) into eq. (7), followed by the substitution of eq. (12). We estimate $\left(\langle n\rangle / n_{0}\right)$ for the case $q E L>>k_{B} T$, i.e., the injection from the drain is neglected. For $E=500 \mathrm{~V} / \mathrm{cm}$ and $L=2 \mu \mathrm{m}$, we have $\left(\langle n\rangle / n_{0}\right)=0.19$. The deficit of carriers is marked for a large $L$, and is mitigated when $L$ decreases. The deficit of the carrier charge in the channel may destroy the charge neutrality of the system. Balancing the carrier charge with the doped impurity charge requires an increase in current injection, which is fulfilled by decreasing the potential barrier of the source-channel 
junction and effectively increasing the carrier density there. The self-consistent Poisson equation will establish some renormalized potential profile. For a low doping concentration $n_{0}$, however, the potential curvature regulated by the Poisson equation is small, and the potential profile approaches the constant field curve when $L$ is sufficiently small.

In an actual system with a large channel length, the presence of energy relaxation due to optical phonon scattering markedly changes the aspect of transport, as is analyzed in Part II. Model 1 with elastic scattering plays an important role in a small system with a very short channel length, exemplified in nanoscale conductors. If energy relaxation is suppressed by some way or another, and yet the elastic scattering is emphasized in the system, the characteristic field dependence proportional to $\sqrt{E}$ may be observed.

We have used the pseudo-one-dimensional model, and it is based on the assumption that the longitudinal kinetic energy and transverse kinetic energy are separately conserved on average in elastic scattering, as discussed in Appendix B. In actuality, however, the incremental gain in longitudinal kinetic energy from the longitudinal field is gradually transferred to the transverse motion toward equilibrium, where the kinetic energy in each direction is equal. The extreme case of close energy exchange can be roughly estimated by assuming that only one-third of the kinetic energy gain from the longitudinal field is used for the acceleration of the longitudinal motion on average. This result, which is estimated from eqs. (5a) and (5b), where $E$ is replaced by $(E / 3)$, requires the horizontal shift by $\ln (3)$ of the curves shown in Figs. 4 and 5. Since this is an extreme case, one can recognize that the qualitative features of the result are not damaged by considering the three-dimensional effect.

\section{Conclusions}


A simple theory of flux equation based on the pseudo-one-dimensional BTE is developed to analyze high-field transport in semiconductors at room temperature. Without relying on RTA or perturbation expansion, we solved BTE and obtained a solution consistent from the low-field to high-field regions.

At a sufficiently low electric field of less than $\sim 500 \mathrm{~V} / \mathrm{cm}$, a carrier's energy is fully relaxed along the channel without yielding hot carriers, and the ordinary drift-diffusion current applies. When the applied field is increased over the limit, the full-energy-relaxation model fails and kinetic energy increases toward the drain. The local quasi-equilibrium collapses and the conventional current model is left groundless. As an extreme instance, the transport only with elastic scattering is first analyzed as Model 1. The transport property is discussed using the transmission coefficient from the source to the drain, $\bar{T}=\tau_{c} /\left(\tau_{c}+\Delta t_{0 \rightarrow L}^{\varepsilon}\right)$. Both the carrier density and the carrier velocity distributions are inhomogeneous within the channel, and current density depends on $E$ and $L$ as $I \propto \sqrt{E / L}$ in the wide field-range of $k_{B} T / q L<<E<<2 m B^{2} L / q$. Carrier flow is hindered for a large $L$, and Ohm's law is shown to break down without energy relaxation.

For an extremely large field of $2 m B^{2} L / q<<E$, the transmission coefficient of carriers through the channel approaches unity, and the ballistic or total transmission results. Electric current tends to saturate at a value given by the thermal injection of carriers from the source into the channel multiplied by the elementary charge.

The extracted transport characteristics may be useful for analysis of an ultrasmall system, e.g., nanoscale devices in which an efficient energy relaxation due to optical phonon scattering is not dominant. On the basis of the present results, in Part II we will analyze the carrier transport that is far from the quasi-equilibrium and suffers from energy relaxation due 
to optical phonon scattering.

\section{Acknowledgement}

The author thanks A. Natori for her continuous support throughout the investigation.

\section{Appendix A: Full-Energy-Relaxation Model and Drift-Diffusion Current}

Here, we develop a simple flux theory in the reflection-transmission probability regime based on the equation of continuity, and apply it to the carrier transport in room-temperature nondegenerate semiconductors. At a low applied field, the carrier energy gained from the field is fully relaxed by carrier scattering without generation of hot carriers, and the mean carrier energy after collision is reduced to thermal energy. We analyze the one-dimensional carrier transport through the channel of length $L$, interposed between the source and drain electrodes. A constant electric field $E$ is assumed in the channel. The positive charge carriers are assumed for simplicity. The $x$-axis is along the channel direction, and the origin is at the source edge. Carriers in the channel can be classified into the positive- and negative-velocity components, and the components constitute each carrier flux. Magnitude of the two fluxes are respectively denoted by $j_{+}(x)$ and $j_{-}(x)$. These fluxes exchange carriers through carrier scattering, and the quantity of exchange is proportional to the carrier density of the flux provided by $j_{ \pm}(x) / v_{ \pm}$, where $v_{+}$and $v_{-}$are the magnitudes of the mean carrier velocity of the positiveand negative-velocity fluxes, respectively. The conservation of each flux in a steady state is formulated. The net density increment is suppressed by the balance of steady flows of the out-scattering to and the in-scattering from the opposite velocity component. The equations of continuity for the two flux components, $j_{+}(x)$ and $j_{-}(x)$, are respectively arranged as 


$$
\begin{aligned}
& \frac{d j_{+}(x)}{d x}+\frac{1}{2 \tau}\left[\frac{j_{+}(x)}{v_{+}}-\frac{j_{-}(x)}{v_{-}}\right]=0, \\
& -\frac{d j_{-}(x)}{d x}+\frac{1}{2 \tau}\left[\frac{j_{-}(x)}{v_{-}}-\frac{j_{+}(x)}{v_{+}}\right]=0,
\end{aligned}
$$

where the probability of scattering to the opposite velocity state is denoted by $1 /(2 \tau)$ and is assumed to be equal for the two flux components [ $\tau$ corresponds to the three-dimensional scattering time. See eq. (B6)]. Equations (A1a) and (A1b) are equivalent to the McKelvey's flux equations ${ }^{13-15)}$. Taking the sum of eqs. (A1a) and (A1b), one can confirm that the net flux $\left[j_{+}(x)-j_{-}(x)\right]$ flowing from the source to the drain is a constant. By combining the result and the difference of these two equations, one can easily solve the pair equation. The solution satisfying the two boundary conditions $j_{+}(0)=j_{+0}$ and $j_{-}(L)=j_{-L}$ is derived as,

$$
\begin{aligned}
& j_{+}(x)=\frac{\left(j_{+0} B_{+}-B_{-} j_{-L}\right) \exp \left[\left(B_{-}-B_{+}\right) x\right]+B_{-}\left\{j_{-L}-j_{+0} \exp \left[\left(B_{-}-B_{+}\right) L\right]\right\}}{B_{+}-B_{-} \exp \left[\left(B_{-}-B_{+}\right) L\right]} \\
& j_{-}(x)=\frac{\left(j_{+0} B_{+}-B_{-} j_{-L}\right) \exp \left[\left(B_{-}-B_{+}\right) x\right]+B_{+}\left\{j_{-L}-j_{+0} \exp \left[\left(B_{-}-B_{+}\right) L\right]\right\}}{B_{+}-B_{-} \exp \left[\left(B_{-}-B_{+}\right) L\right]},
\end{aligned}
$$

where $B_{ \pm} \equiv 1 /\left(2 \tau v_{ \pm}\right)$. Owing to the full energy relaxation, the average energy of a carrier immediately after the scattering is the thermal energy $k_{\mathrm{B}} T$ at any point in the channel; therefore, mean velocity is reduced to the thermal velocity $v_{0}=\sqrt{2 k_{B} T / \pi m}$ for both the positive- and negative-velocity fluxes. The velocity increment due to the electric field immediately before the next scattering is $2 \tau \cdot q E / m$, whose magnitude is far smaller than $v_{0}$ for the assumed low field. The relevant energy increment also is much smaller than the thermal energy, and it is relaxed by the acoustic phonon scattering. Then, the mean carrier velocities for both fluxes are 


$$
v_{ \pm}=v_{0} \pm \frac{q E \tau}{m}
$$

and so $v_{-} \approx v_{+} \approx v_{0}$. One can assume

$$
\left(B_{-}-B_{+}\right) L \approx \frac{q E L}{m v_{0}^{2}}>>1 \quad \text { if } \quad V \equiv E L>>k_{B} T / q .
$$

The terms with exponential factors are dominant in eqs. (A2a) and (A2b), and we notice $j_{+}(x) \approx j_{-}(x) \approx j_{+0}$ within a wide range of $0 \leq x<<\left[L-1 /\left(B_{-}-B_{+}\right)\right]$even if $j_{+0} \neq j_{-L}$, in accordance with the assumption of homogeneity in eq. (1). The carrier density $n(x)$ is given by

$$
n(x)=\frac{j_{+}(x)}{v_{+}}+\frac{j_{-}(x)}{v_{-}} .
$$

Equations (A1a), (A1b), and (A5), as well as $d n(x) / d x$ derived from eq. (A5), yield the expressions of $j_{+}(x)$ and $j_{-}(x)$ in terms of $n(x)$ and $d n(x) / d x$. Then the electric current density $I=q\left\{j_{+}(x)-j_{-}(x)\right\}$ is transformed to

$$
I=\frac{1}{2} q n(x)\left(v_{+}-v_{-}\right)-q v_{+} v_{-} \tau \frac{d n(x)}{d x}=q n \frac{q \tau}{m} E-v_{0}^{2} \tau \frac{d n(x)}{d x} .
$$

Since $v_{0}{ }^{2} \tau$ is the diffusion coefficient for room temperature, eq. (A6) correctly reproduces drift-diffusion current. Equations (A2a) and (A2b) yield both the transmission coefficient $\bar{T}$ and the reflection coefficient $\bar{R}$. They are values of $j_{+}(L)$ and $j_{-}(0)$ for the simultaneous boundary values $j_{+0}=1$ and $j_{-L}=0$. We obtain $\bar{T} \approx 2 q \tau E / m v_{0}$ for drift current or $\bar{T} \approx 2 v_{0} \tau / L$ for diffusion current $\left(q E L<<m v_{0}{ }^{2}\right)$, and $\bar{T}<<1$ and $\bar{R} \sim 1$ in either case.

We next estimate the electric field range in which the full energy relaxation approximation is valid. Since the mean carrier velocity is roughly $\left(v_{+}-v_{-}\right) / 2=(q \tau / m) E$, the mean energy 
gain of a carrier during the scattering time $2 \tau$ is roughly estimated as $q E \cdot(q \tau / m) E \cdot 2 \tau$. We then estimate the mean energy relaxation of a carrier. The major inelastic processes are optical phonon scattering and acoustic phonon scattering. The former involves a large quantity of energy and is the efficient route to energy relaxation. However, it is not dominant at low applied fields, at which the relevant carrier energy is small. Acoustic phonon scattering, which is sometimes treated as elastic, involves a small quantity of energy, and the energy relaxation due to the process is dominant in sufficiently low field transport. The energy exchanged by the acoustic phonon scattering is a few milli-eV for thermal electrons, and we assume it is $\sim 0.1 k_{\mathrm{B}} T$ at room temperature. The emission probability of the phonon for an electron is proportional to $(\langle N\rangle+1)$ and the absorption to $\langle N\rangle$, where $\langle N\rangle=1 /[\exp (0.1)-1]=9.5$ is the mean number of phonons. Thus, the mean energy relaxation per acoustic phonon scattering is $0.1 k_{\mathrm{B}} T \times(10.5-9.5) /(10.5+9.5)=0.005 k_{\mathrm{B}} T$, where the thermal energy is $k_{\mathrm{B}} T=0.026 \mathrm{eV}$ at room temperature. Therefore, the hypothesis of full energy relaxation is validated if

$$
2 q\left(\frac{q \tau E}{m} \tau\right) E \leq 0.005 k_{B} T
$$

which yields $E \leq 490 \mathrm{~V} / \mathrm{cm}$ as the validity range of the full-energy-relaxation model, if we employ the parameters presented in $\S 2$.

\section{Appendix B: Derivation of a Pseudo-one-dimensional Model from the}

\section{Three-Dimensional Boltzmann Transport Equation}

The pseudo-one-dimensional model used in the analysis is derived from the three-dimensional steady-state BTE characterized by the spherical effective mass $m$ 


$$
\frac{q}{\hbar} \mathbf{E} \cdot \frac{\partial f_{\mathbf{r}, \mathbf{k}}}{\partial \mathbf{k}}+\frac{\hbar \mathbf{k}}{m} \cdot \frac{\partial f_{\mathbf{r}, \mathbf{k}}}{\partial \mathbf{r}}-\int\left[f_{\mathbf{r}, \mathbf{k}^{\prime}}\left(1-f_{\mathbf{r}, \mathbf{k}}\right)-f_{\mathbf{r}, \mathbf{k}}\left(1-f_{\mathbf{r}, \mathbf{k}^{\prime}}\right)\right] Q_{\mathbf{r}, \mathbf{k}, \mathbf{k}} \cdot \frac{d \mathbf{k}^{\prime}}{(2 \pi)^{3}}=0
$$

Here, $f_{\mathbf{r}, \mathbf{k}}$ is the three-dimensional distribution function and $Q_{\mathbf{r}, \mathbf{k}, \mathbf{k}^{\prime}}$ is the transition probability between the initial and final states. Abbreviated expressions for the differentiation and the integration by vectors are used. Elastic scattering is assumed. As usual for the room-temperature Boltzmann statistics, we assume that the carrier distribution is dilute and (1 $\left.-f_{\mathrm{r}, \mathbf{k}}\right) \approx 1$. We use cylindrical coordinates $\left(k_{\mathrm{x}}, k_{\perp}, \theta\right)$ in place of $\left(k_{\mathrm{x}}, k_{\mathrm{y}}, k_{\mathrm{z}}\right)$. We assume a device structure where the electric field $\mathbf{E}$ with a magnitude $E$ is applied parallel to the $x$-axis of a homogeneous sample. The system is homogeneous in the $y$-z-plane and has a cylindrical symmetry around the $x$-axis. Then, the dependence of the distribution function $f_{\mathbf{r}, \mathbf{k}}$ on the coordinates $y, z$, and $\theta$ is eliminated, and we have $f_{\mathbf{r}, \mathbf{k}}=f\left(x, k_{x}, k_{\perp}\right)$. Equation (B1) is rewritten as

$$
\begin{aligned}
& \frac{q}{\hbar} E \\
& \quad \frac{\partial f\left(x, k_{x}, k_{\perp}\right)}{\partial k_{x}}+\frac{\hbar k_{x}}{m} \frac{\partial f\left(x, k_{x}, k_{\perp}\right)}{\partial x} \\
& \quad+\int\left[f\left(x, k_{x}, k_{\perp}\right)-f\left(x, k_{x}{ }^{\prime}, k_{\perp}{ }^{\prime}\right)\right] Q\left(x, k_{x}, k_{\perp}, \theta, k_{x}{ }^{\prime}, k_{\perp}{ }^{\prime}, \theta^{\prime}\right) \frac{d k_{x}{ }^{\prime} k_{\perp}{ }^{\prime} d k_{\perp}{ }^{\prime} d \theta^{\prime}}{(2 \pi)^{3}}=0 .
\end{aligned}
$$

To reduce eq. (B2) to a pseudo-one-dimensional expression along the $x$-axis, some averaging of the collision part over $k_{\perp}$ in the $k_{\mathrm{y}}-k_{\mathrm{z}}$ plane is inevitable. Elastic scattering requires $\hbar^{2}\left(k_{x}{ }^{2}+k_{\perp}{ }^{2}\right) / 2 m=\hbar^{2}\left(k_{x}{ }^{2}+k_{\perp}{ }^{\prime 2}\right) / 2 m$. The longitudinal energy $\hbar^{2} k_{x}{ }^{2} / 2 m$ is varied by $E$ and also by the scattering. The transverse energy $\hbar^{2} k_{\perp}{ }^{2} / 2 m$ is varied only by the scattering, and is increased or decreased by interaction with the longitudinal energy. We assume that the increase and the decrease occur roughly by equal chance. Thus, we can require that $\hbar^{2} k_{\perp}{ }^{2} / 2 m \approx \hbar^{2} k_{\perp}{ }^{2} / 2 m$ on average. So, we employ an approximate expression of scattering probability in terms of delta functions 


$$
Q\left(x, k_{x}, k_{\perp}, \theta, k_{x}{ }^{\prime}, k_{\perp}{ }^{\prime}, \theta^{\prime}\right) \cong Q \delta\left[\frac{\hbar^{2}\left(k_{x}{ }^{2}+k_{\perp}{ }^{2}\right)}{2 m}-\frac{\hbar^{2}\left(k_{x}{ }^{2}+k_{\perp}{ }^{2}\right)}{2 m}\right] \delta\left(\frac{\hbar^{2} k_{\perp}{ }^{2}}{2 m}-\frac{\hbar^{2} k_{\perp}{ }^{2}}{2 m}\right)
$$

Physically, it is a stronger restriction that the longitudinal and transverse energies should be separately conserved, compared with the actual condition that only the sum of these energies is conserved. $Q$ generally depends on $x, k_{\mathrm{x}}, k_{\perp}$, and $\left(\theta-\theta^{\prime}\right)$. However, we expect a simple scattering model here and neglect the $x, k_{\perp}$, and $\left(\theta-\theta^{\prime}\right)$ dependence of $Q$, assuming appropriate averaging over these parameters. Substituting eq. (B3) into eq. (B2), the collision integral part is transformed to

$$
\frac{1}{(2 \pi)^{2}} \frac{m^{2}}{\hbar^{4}} Q \frac{1}{\left|k_{x}\right|}\left[f\left(x, k_{x}, k_{\perp}\right)-f\left(x,-k_{x}, k_{\perp}\right)\right]
$$

On the other hand, the scattering time $\tau$ in our case is evaluated using eq. (B3) as

$$
\frac{1}{\tau}=\int Q\left(x, k_{x}, k_{\perp}, \theta, k_{x}{ }^{\prime}, k_{\perp}{ }^{\prime}, \theta^{\prime}\right) \frac{d k_{x}{ }^{\prime} k_{\perp}{ }^{\prime} d k_{\perp}{ }^{\prime} d \theta^{\prime}}{(2 \pi)^{3}}=\frac{2}{(2 \pi)^{2}} \frac{m^{2}}{\hbar^{4}} Q \frac{1}{\left|k_{x}\right|} .
$$

Integrating both sides of eq. (B2) over the plane of $k_{\perp}$ and $\theta$ at a given $k_{\mathrm{x}}$, while also summing over the spin, and using eq. (B5), we obtain a one-dimensional BTE

$$
\frac{q}{\hbar} E \frac{\partial f\left(x, k_{x}\right)}{\partial k_{x}}+\frac{\hbar k_{x}}{m} \frac{\partial f\left(x, k_{x}\right)}{\partial x}+\frac{1}{2 \tau}\left[f\left(x, k_{x}\right)-f\left(x,-k_{x}\right)\right]=0 .
$$

Here, $f\left(x, k_{\mathrm{x}}\right)$ is the pseudo-one-dimensional distribution function in the $x$-direction given by

$$
f\left(x, k_{x}\right) \equiv 2 \int f\left(x, k_{x}, k_{\perp}\right) \frac{k_{\perp} d k_{\perp} d \theta}{(2 \pi)^{2}} .
$$

The three-dimensional carrier density is derived by

$$
\int f\left(x, k_{x}\right) \frac{d k_{x}}{2 \pi}
$$


and the three-dimensional flux of the structure is given by

$$
\int f\left(x, k_{x}\right) \frac{\hbar k_{x}}{m} \frac{d k_{x}}{2 \pi}
$$

For the inelastic optical phonon emission discussed in Part II as well, we assume that the transverse energy is kept unaltered and consequently derive a one-dimensional BTE similar to eq. (B6). We assume that the electric field increases, the elastic scattering conserves, and the optical phonon emission decreases longitudinal energy alone, keeping transverse energy unaltered, thus constituting the pseudo-one-dimensional model.

To calculate current density, we evaluate the flux injected into the sample from a quasi-equilibrium reservoir with the carrier density $n_{0}$. The three-dimensional distribution function of the reservoir is approximated by the Boltzmann statistics with the Fermi energy $\mu$ as

$$
f_{\text {res }}\left(x, k_{x}, k_{\perp}\right)=\exp \left[\left(\mu-\frac{\hbar^{2} k_{x}^{2}+\hbar^{2} k_{\perp}^{2}}{2 m}\right) \frac{1}{k_{B} T}\right] \text {. }
$$

Then, eq. (B7) yields

$$
f_{\text {res }}\left(x, k_{x}\right)=\frac{m k_{B} T}{\pi \hbar^{2}} \exp \left[\left(\mu-\frac{\hbar^{2} k_{x}^{2}}{2 m}\right) \frac{1}{k_{B} T}\right] .
$$

and expression (B8) yields

$$
n_{0}=\frac{1}{\sqrt{2}}\left(\frac{m k_{B} T}{\pi \hbar^{2}}\right)^{3 / 2} \exp \left(\frac{\mu}{k_{B} T}\right)
$$

The injected flux with longitudinal carrier energy between $\varepsilon$ and $(\varepsilon+\mathrm{d} \varepsilon)$ is evaluated using eq. (B12) as 


$$
f_{\text {res }}\left(x, k_{x}\right) \frac{\hbar k_{x}}{m}\left(\frac{1}{2 \pi} \frac{d k_{x}}{d \varepsilon_{x}}\right) d \varepsilon_{x}=\frac{n_{0}}{\sqrt{2 \pi m k_{B} T}} \exp \left(-\frac{\varepsilon_{x}}{k_{B} T}\right) d \varepsilon_{x} \text {. }
$$


1) E. J. Ryder: Phys. Rev. 90 (1953) 766.

2) W. Shockley: Bell Syst. Tech. J. 30 (1951) 990.

3) W. Shockley: Electrons and Holes in Semiconductors (Van Nostrand, Princeton, NJ, 1950).

4) J. M. Ziman: Principles of the Theory of Solids (Cambridge University Press, Cambridge, U.K. 1972) 2nd ed.

5) H. U. Baranger and J. W. Wilkins: Phys. Rev. B 30 (1984) 7349.

6) A. A. Ignatov, E. P. Dodin, and V. L. Shashkin: Mod. Phys. Lett. B 5 (1991) 1087.

7) G. D. Mahan: J. Appl. Phys. 58 (1985) 2242.

8) T. Kurosawa: Proc. Int. Conf. Physics Semiconductors, Kyoto, 1966, J. Phys. Soc. Jpn. 21 (1966) Suppl., p. 424.

9) C. Jacoboni and L. Reggiani: Rev. Mod. Phys. 55 (1983) 645.

10) M. V. Fischetti and S. E. Laux: Phys. Rev. B 48 (1993) 2244.

11) S. Datta: Electronic Transport in Mesoscopic Systems (Cambridge University Press, Cambridge, U.K., 1995) p. 57.

12) R. Landauer: Phys. Scr. T42 (1992) 110.

13) J. P. McKelvey, R. L. Longini, and T. P. Brody: Phys. Rev. 123 (1961) 51.

14) J. H. Rhew and M. S. Lundstrom: J. Appl. Phys. 92 (2002) 5196.

15) G. Gildenblat: J. Appl. Phys. 91 (2002) 9883.

16) R. Clerc, P. Palestri, and L. Selmi: IEEE Trans. Electron Devices 53 (2006) 1634.

17) F. J. Blatt: in Solid State Physics, ed. F. Seitz and D. Turnbull (Academic, New York, 1957) vol. 4.

18) P. Drude: Ann. Phys. (Leipzig) 1 (1900) 566 [in German]. 
19) N. W. Ashcroft and N. D. Mermin: Solid State Physics (Saunders College, Philadelphia, PA, 1976).

20) K. Seeger: Semiconductor Physics (Springer, Berlin, 1989).

21) E. M. Conwell: in High Field Transport in Semiconductors, ed. F. Seitz, D. Turnbull, and H. Ehrenreich (Academic, New York, 1967).

22) K. Natori and T. Shimizu: Ext. Abstr. Solid State Devices and Materials, 2006, p. 348.

23) K. Natori: Phys. Status Solidi C 5 (2008) 111.

24) K. Natori: submitted to Jpn. J. Appl. Phys.

25) For example, M. Levinshtein, S. Runyanstev, and M. Shur: Handbook Series on Semiconductor Parameters (World Scientific, Singapore, 1996) Vol. 1, p.1. 


\section{Figure Captions}

Fig. 1. Potential profile of the device and carrier transport accompanied by the energy-conserving elastic scattering along the channel (Model 1). The kinetic energy of the carrier increases along the channel owing to energy gain from the field, leading to hot carrier generation.

Fig. 2. Distribution of carrier density along the channel for various values of $L$. The applied field is $1000 \mathrm{~V} / \mathrm{cm}$, and the incident kinetic energy $\varepsilon$ is the thermal energy $0.026 \mathrm{eV}$ at room temperature. Value 2, shown as the carrier density at $x=0$, implies that the incident flux and the flux reflected into the source have the same magnitude.

Fig. 3. Distribution of drift velocity (mean velocity) along the channel for various values of $L$. The applied field is $1000 \mathrm{~V} / \mathrm{cm}$, and $\varepsilon$ is $0.026 \mathrm{eV}$. The value of velocity is normalized by the incident carrier velocity. The dashed line indicates the value given by the conventional mobility multiplied by the field.

Fig. 4. Transmission coefficient from source to drain as a function of the applied field for various values of $L$.

Fig. 5. Current density from source to drain as a function of the field for various values of $L$. 


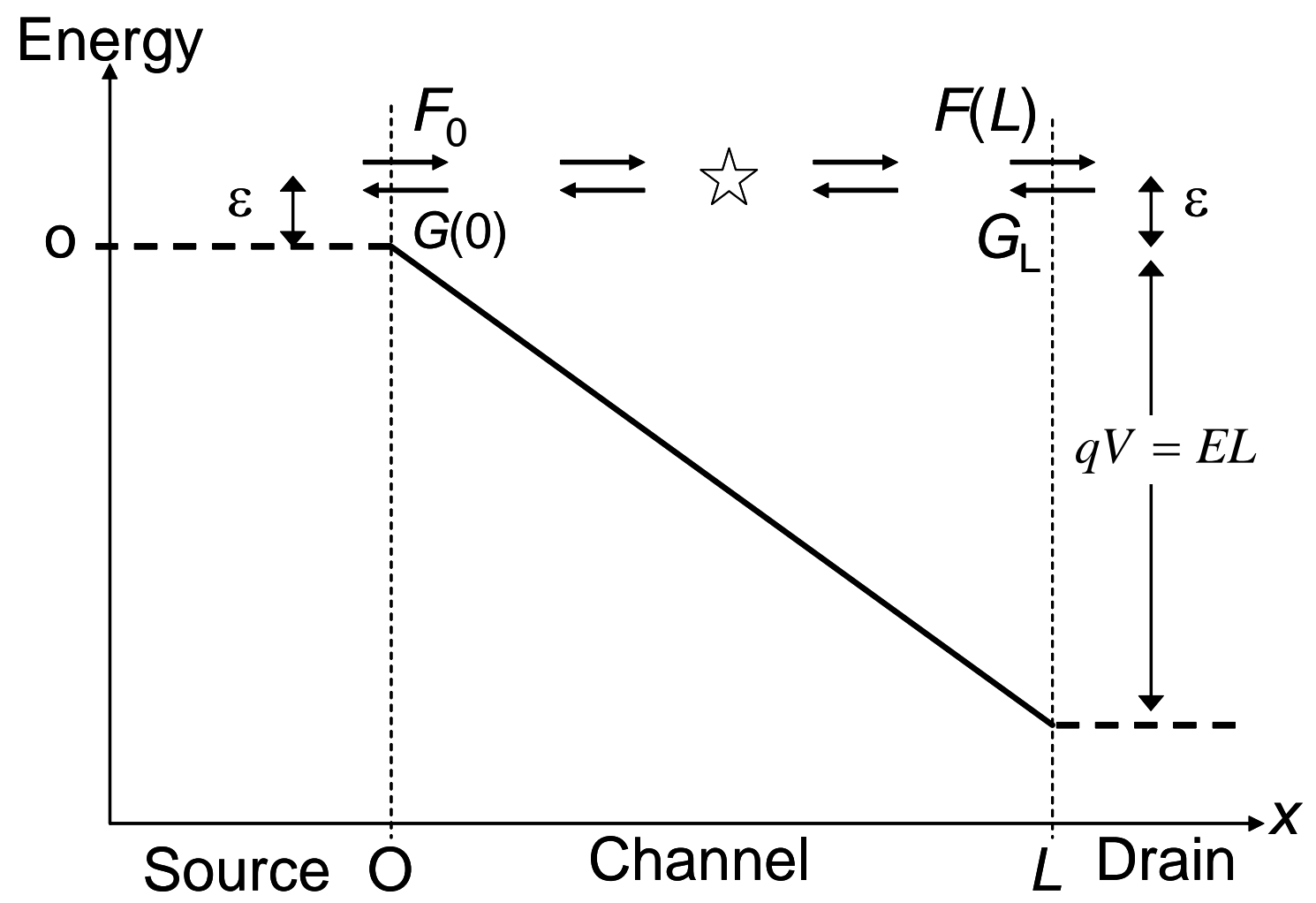

Fig. 1 


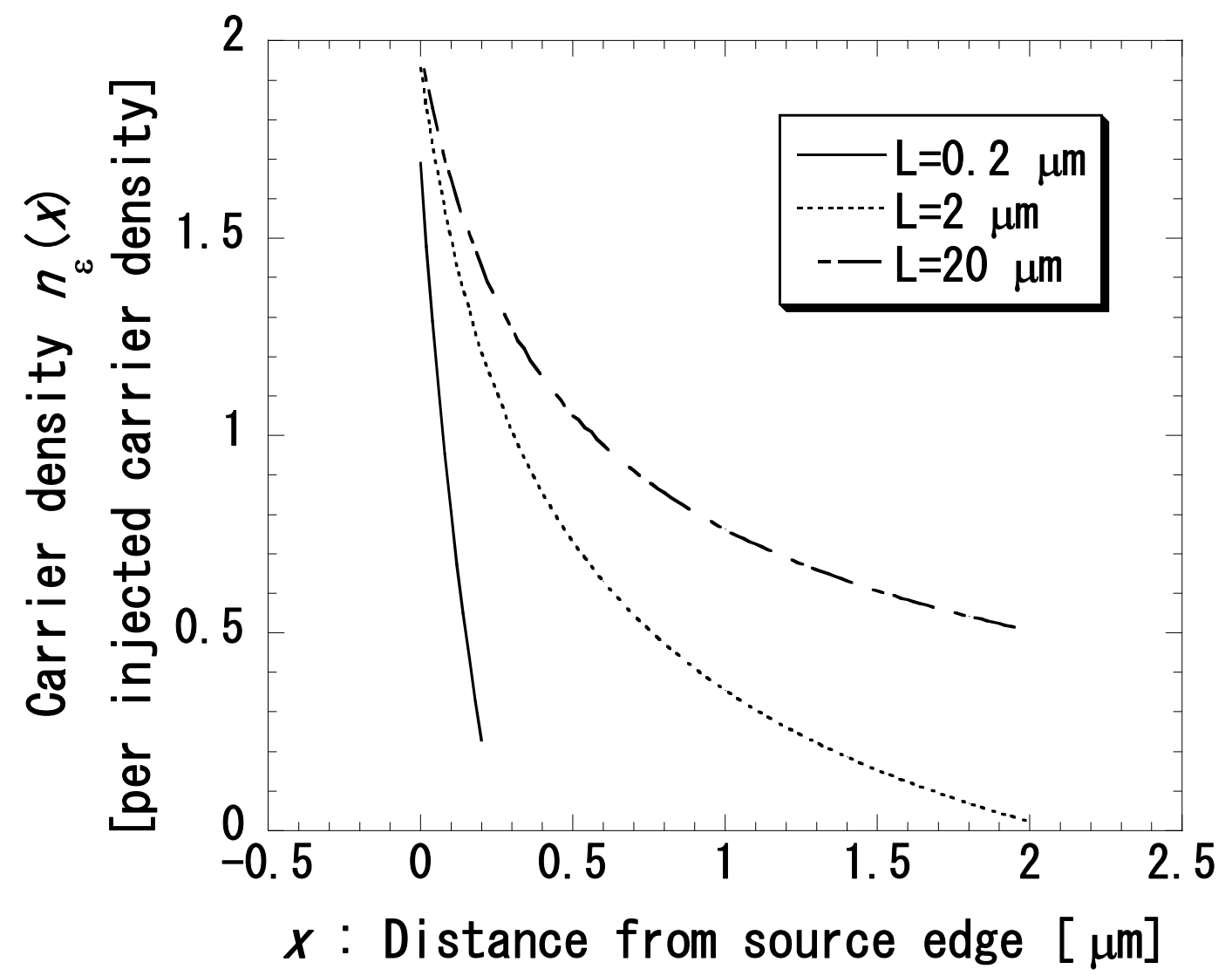

Fig. 2 


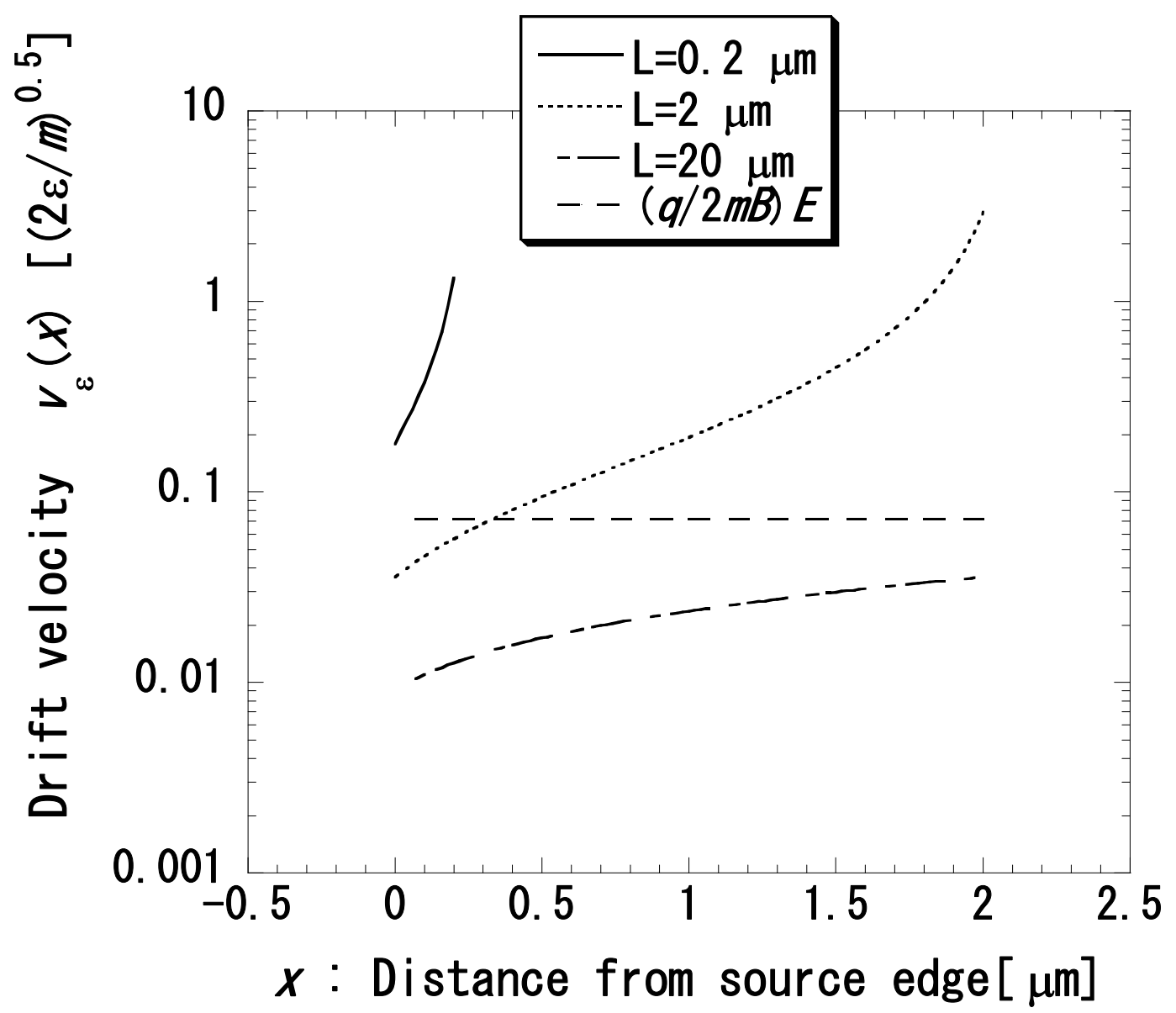

Fig. 3 


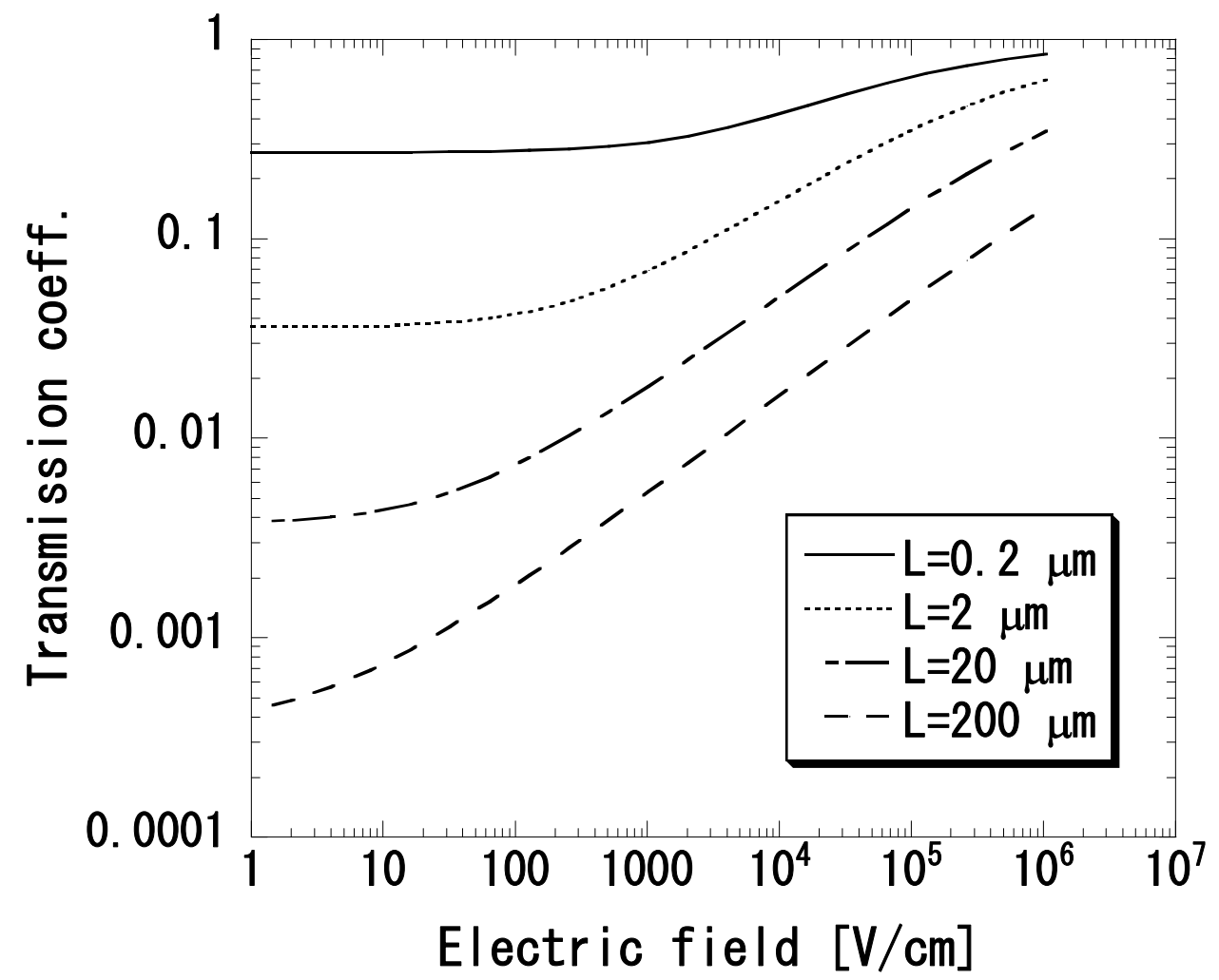

Fig. 4 


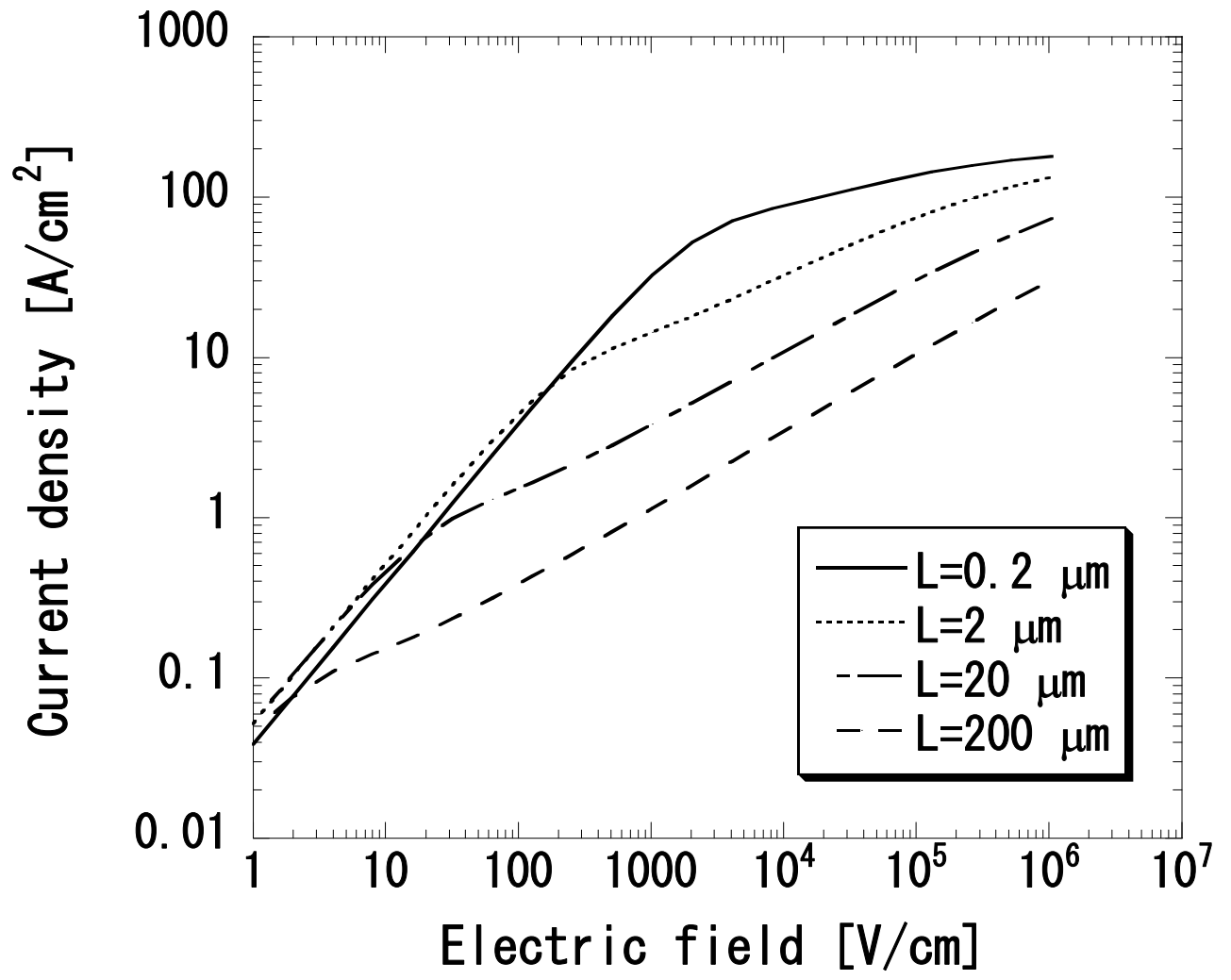

Fig. 5 